\title{
Evaporation Modelling for Low-temperature, Long-term Exposure of Wood under Isothermal Heating
}

\author{
SIEW MEI LIM ${ }^{1}$ and MICHAEL YIT LIN CHEW ${ }^{2}$ \\ ${ }^{1}$ Rolf Jensen \& Associates, Inc., One Pointe Drive, Suite 210, Brea, CA 92821 USA \\ ${ }^{2}$ Department of Building, National University of Singapore, 4, Architecture Drive, School of Design \\ and Environment, National University of Singapore, Singapore 117566
}

\begin{abstract}
This research studied the combustion of wood exposed to low-temperature for an extended duration. A new evaporation approach was developed for low-temperature extended heating of wood. Evaporation was modeled as a boundary condition for initial drying, and internal evaporation term was re-introduced for extended heating model. A porous model was developed through the use of effective transport properties that enabled a reliable representation of heterogeneous medium of wood to be achieved by a homogenous model. Darcy's law was modified to account for inertia and boundary effects on the flows in a porous structure. The numerical model showed that the free water movement produced constant temperature distribution for initial phase. As heating continued, the transport of combined moisture was strongly coupled with temperature field development. The numerical solution illustrated the importance of the interaction of moisture flows and temperature development for self-heating.
\end{abstract}

KEYWORDS: self-heating, modeling, low-temperature, long-term heating, wood, evaporation.

\section{NOMENCLATURE LISTING}

\begin{tabular}{llll}
$c$ & specific heat $(\mathrm{J} / \mathrm{kg})$ & $\mathrm{V}$ & vapour \\
$D$ & effective diffusivity $\left(\mathrm{m} / \mathrm{s}^{2}\right)$ & $v$ & Sample volume $\left(\mathrm{m}^{3}\right)$ \\
$h$ & heat transfer coefficient $\left(\mathrm{W} / \mathrm{m}^{2} \mathrm{k}\right)$ & $\mathrm{W}$ & water \\
$\Delta H_{e v}$ & heat of evaporation $(\mathrm{J} / \mathrm{mol})$ & $x$ & dimension $(\mathrm{m})$ \\
$h_{D}$ & mass transfer coefficient $\left(\mathrm{kg} / \mathrm{m}^{2} \mathrm{~s}\right)$ & \multicolumn{2}{c}{ Greek } \\
$I$ & evaporation term & $\varepsilon$ & emissivity \\
$k$ & thermal conductivity $(\mathrm{W} / \mathrm{mK})$ & $\varphi$ & porosity \\
$M$ & moisture flux $(\mathrm{m} / \mathrm{s})$ & $\rho$ & density $\left(\mathrm{kg} / \mathrm{m}^{3}\right)$ \\
$P$ & pressure $\left(\mathrm{N} / \mathrm{m}^{2}\right)$ & subscripts \\
$q$ & heat flux $\left(\mathrm{kW} / \mathrm{m}^{2}\right)$ & $e$ & external \\
$R$ & universal gas constant $\left(8.314 \mathrm{~J} \mathrm{~K}^{-1} \mathrm{~mol}^{-1}\right)$ & $e v \quad$ evaporation \\
$R_{e v}$ & rate of evaporation & $s$ & solid \\
$T$ & temperature $(\mathrm{K})$ & $s a t$ & saturation \\
$T_{\infty}$ & ambient temperature $(\mathrm{K})$ & $m$ & moisture \\
$t$ & time $(\mathrm{s})$ & $v$ & vapour \\
$u$ & velocity $(\mathrm{m} / \mathrm{s})$ & $w$ & water
\end{tabular}

\section{INTRODUCTION}

The heating of wood brings upon physical changes such as enthalpy and moisture content. One of the major links between temperature and moisture changes is water evaporation. Water evaporation acts as a heat source term in energy balance, and contributes to physical processes of heat and mass transfer in the heating of wood. In most of the work on mass transfer, the onset of convection in porous media (with or without chemical reaction) has assumed the fluid in the basic equilibrium state is motionless [1]. However, the presence of both thermal and concentration effects as well as the existence of fluid density gradients can eventually induce natural convection of fluid. Therefore, evaporation other than acting as an endothermic heat of reaction, its resulting moisture movement also plays a critical role in the spontaneous heating of wood [2].

High temperature drying model does not typically consider liquid water transport, and moisture flow takes place essentially in vapour phase [3-5]. However, the true mechanisms of moisture transport for wood drying, where the moisture content of wood varies between free water continuity and fiber saturation point, 
are still open for discussion. Perré at al [6] successfully modelled both liquid and gaseous transport while Krabbenhøft [7] pointed out that there is certain justification for assuming transport of free water under partially saturated conditions. In low temperature drying model of wood, there is a stronger reason to restore free water movement since drying would be slower and there would be measurable movement of free water during this stage.

It remains a challenge to represent a heterogeneous medium of wood as a homogeneous model for a more tractable mathematical treatment on moisture transport. Vortmeyer, Dietrich and Ring [8] pointed out that a reliable representation of a heterogeneous media can be achieved by a homogeneous model with the introduction of effective transport coefficients. The effective transport properties, introduced through the combined moisture flow approach, enable the wood slab to at least attain pseudo-homogeneity. It then remains a question of fact if it is realistic to combine the moisture flows in the case of low-temperature heating of wood. In high temperature drying model, the moisture flow was normally divided into vapour flow and water flow [9]. This assumption is endorsed on the basis that for drying associated with rapid heating, heat flow is different from the total energy flow. There is however no reason for low-temperature drying model to divide the moisture flows. Further, recent research has shown that the combined approach produced satisfactory results for high-temperature drying [10,11], as well as low-temperature drying [12].

In this study of evaporation modelling for wood drying at low temperature, a combined approach on moisture flow is adopted to achieve homogeneity, which is more accurate in the description of the moisture flow for the low-drying regime in wood. A different description of moisture evaporation was used to model wood drying at low temperature. The proposed low-temperature evaporation model seeks to address some problems associated with low-temperature combustion phenomenon by firstly restoring moisture for low temperature heating where high temperature evaporation model would have rapidly vaporized the moisture; secondly redressing the importance of moisture flow at low temperature regime which has been surpassed by high temperature heating model.

\section{EVAPORATION MODEL FORMULATION}

The heat and mass transfer model in the porous slab is modelled as one-dimensional flow where it could be schematically represented in Fig. 1. The anisotropy and wood geometry are illustrated in the experimental section.

Exposed surface Insulated surface

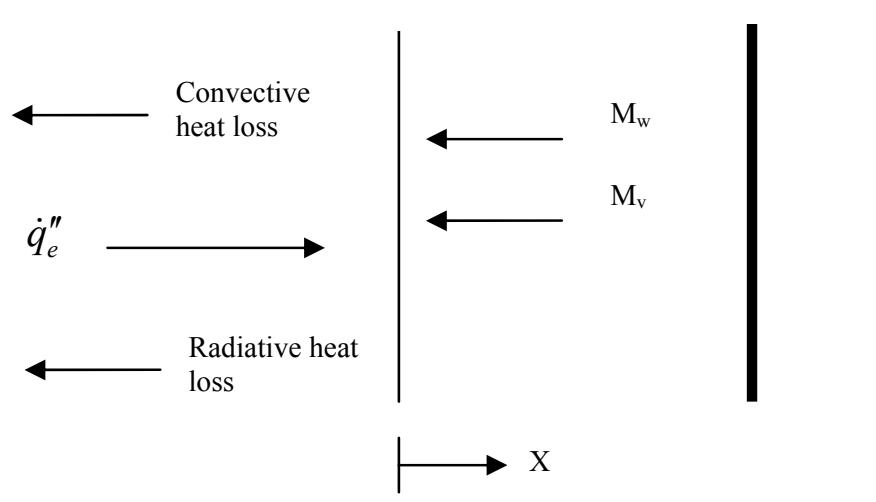

Fig. 1. The one-dimensional flow in porous model

The following assumptions are introduced alongside the development of the models in order to make the mathematical treatment tractable:

- Wood is modeled as a porous slab. However, all structural changes such as swelling, shrinkage, crack formation, are negligible during drying. 
- Liquid and gaseous transport is dominant and responsible for internal heat transfer, through diffusion and convection.

- Pyrolysis is negligible at low temperature drying.

- Water and vapour fluxes are combined into a total moisture flux with effective diffusivity, which includes two phases and two transport mechanisms.

- Evaporation of moisture is sufficiently rapid to attain thermodynamic equilibrium.

- Movement of both water and combined moisture flow are taken into account using Darcy's law.

- Escaping vapour is in thermal equilibrium with the solid matrix. The out flowing vapour does not withdraw sufficient energy from the solid to affect the solid temperature. In other words, the mass flux is slow.

A combined moisture flow of liquid and vapour is introduced for the porous model when the internal evaporation is considered. To combine the liquid and vapour flow, a model for typical water $(W)$ and vapour $(V)$ conservation laws is first considered

$$
\begin{aligned}
& \frac{\partial W}{\partial t}=\frac{\partial}{\partial x}\left(D_{w} \frac{\partial W}{\partial t}\right)+\frac{\partial}{\partial x}\left(\rho_{w} c_{w} v_{w}\right) T-I \\
& \frac{\partial V}{\partial t}=\frac{\partial}{\partial x}\left(D_{V} \frac{\partial V}{\partial t}\right)+\frac{\partial}{\partial x}\left(\rho_{v} c_{v} v_{v}\right) T+I
\end{aligned}
$$

Combining Equations (1) and (2) leads to

$$
\frac{\partial W}{\partial t}+\frac{\partial V}{\partial t}=\frac{\partial}{\partial x}\left(D_{w} \frac{\partial W}{\partial x}\right)+\frac{\partial}{\partial x}\left(D_{v} \frac{\partial V}{\partial x}\right)+\frac{\partial}{\partial x}\left(\rho_{w} c_{w} v_{w}\right) T+\frac{\partial}{\partial x}\left(\rho_{v} c_{v} v_{v}\right) T
$$

where the evaporation term $(I)$ has been eliminated. Total moisture content in mass of water per unit mass of dry matter $(M)$ is defined as

$$
M=(V+W) / \rho_{s}
$$

and a total moisture flux (diffusive and convective) is written as

$$
\frac{\partial M}{\partial t}=\frac{\partial}{\partial x}\left(D_{m} \frac{\partial M}{\partial x}\right)+\frac{\partial}{\partial x}\left(\rho_{m} c_{m} v_{m}\right) T
$$

A new parameter $D_{m}$, known as the effective diffusivity is introduced, combining water diffusivity $D_{w}$ and vapour diffusivity $D_{v}$. The concept of effective transport property lump the two phases and different transport mechanisms together as one. A two-tier model is presented for modelling moisture transport within the porous slab. For the initial heating phase, surface evaporation is considered and evaporation term is eliminated from the heat balance. Evaporation is deemed to occur at evaporation temperature at $100^{\circ} \mathrm{C}$. Extended drying phase is defined when the evaporation front recesses into the domain, as heating continues when the temperature has reached $100^{\circ} \mathrm{C}$. An internal evaporation term is then re-introduced into the heat balance for extended drying phase.

\section{For initial drying phase}

Energy Balance

$$
\frac{\partial}{\partial t}(\overline{\rho c} T)=-\varphi \nabla \cdot\left(\vec{u} \rho_{w} c_{w} T\right)+\nabla \cdot k_{e f f} \cdot \nabla T
$$


where $k_{\text {eff }}$ is the effective thermal conductivity, $(\overline{\rho c})$ is the average heat capacity of the solid and fluid mixture medium. The effective thermal conductivity is defined as

$k_{\text {eff }}=\varphi k_{m}+(1-\varphi) k_{s}$

The average heat capacity is defined as

$\overline{\rho c}=\rho_{s}(1-\varphi) c_{s}+\rho_{m} \varphi c_{m}$

Mass Balance

$\varphi \frac{\partial W}{\partial t}=-\varphi \nabla \cdot\left(M_{w} \vec{u}\right)+\varphi \nabla \cdot D_{w} \cdot \nabla M_{w}$

where the internal term of the rate of evaporation is omitted.

Initial and boundary conditions for energy equation

Boundary condition $(\mathrm{x}=0)$

$-k_{e f f} \frac{\partial T}{\partial x}=\dot{q}_{e}^{\prime \prime}-h\left(T-T_{\infty}\right)-\varepsilon \sigma\left(T^{4}-T_{\infty}^{4}\right)$

Boundary condition $(\mathrm{x} \rightarrow \infty)$

$-k_{\text {eff }} \frac{\partial T}{\partial x}=0$

Initial condition $(\mathrm{t}=0, \mathrm{x} \geq 0)$

$T=T_{\infty}$

Initial and boundary conditions for conservation of mass

Boundary condition $(\mathrm{x}=0)$

$-\rho_{w} D_{w} \frac{\partial W}{\partial x}(0, t)=h_{D}\left(M_{w}(0, t)-M_{w, \infty}(t)\right)$

Boundary condition $(\mathrm{x} \rightarrow \infty)$

$-D_{w} \frac{\partial W}{\partial t}=0$

Initial condition $(t=0, x \geq 0)$

$M_{w, 0}=M_{w, \infty}$

\section{For extended drying phase}

An internal evaporation term is formulated assuming equilibrium approach. The conservation equations are as follows:

Energy Balance

$\frac{\partial}{\partial t}(\overline{\rho c} T)=-\varphi \nabla \cdot\left(\vec{u} \rho_{m} c_{m} T\right)+\nabla \cdot k_{e f f} \cdot \nabla T+(1-\varphi) \rho_{s}\left(-\Delta H_{e v} R_{e v}\right)$

where $k_{\text {eff }}$ is the effective thermal conductivity, $(\overline{\rho c})$ is the average heat capacity of the solid and fluid mixture medium. For total moisture transport, the effective thermal conductivity is defined as 
$k_{\text {eff }}=\varphi k_{m}+(1-\varphi) k_{s}$

The average heat capacity is defined as

$\overline{\rho c}=\rho_{s}(1-\varphi) c_{s}+\rho_{m} \varphi c_{m}$

Mass Balance

$\varphi \frac{\partial M}{\partial t}=-\varphi \nabla \cdot\left(M_{m} \vec{u}\right)+\varphi \nabla \cdot D_{m} \cdot \nabla M_{m}-(1-\varphi) \rho_{s} R_{e v}$

An internal evaporation term is re-introduced into the energy balance and the rate of evaporation also now appears in the mass balance. The initial and boundary conditions for energy conservation are the same and not repeated. The initial and boundary conditions for mass conservation are described below for moisture transport.

\section{Initial and boundary conditions for conservation of mass}

Boundary condition $(\mathrm{x}=0)$

$-\rho_{m} D_{m} \frac{\partial M}{\partial x}(0, t)=h_{D}\left(M_{m}(0, t)-M_{m, \infty}(t)\right)$

Boundary condition $(\mathrm{x} \rightarrow \infty)$

$-D_{m} \frac{\partial M}{\partial t}=0$

Initial condition $(\mathrm{t}=0, \mathrm{x} \geq 0)$

$M_{m, 0}=M_{m, \infty}$

Equilibrium approach is adopted in the low-drying model, and the equilibrium between liquid and vapour is assumed to reach instantaneously, so that

$P_{v}=P_{\text {sat }}(T)$

in the presence of liquid water. A simple analytical expression is used to relate the saturation pressure to temperature [13]. The expression has the following form:

$P_{\text {sat }}(T)=C T^{-B / R_{v}} \exp \left(-\frac{A}{R_{v} T}\right)$

where $A=3.18 \times 10^{6} \mathrm{~J} \mathrm{~kg}^{-1}, B=2500 \mathrm{~J} \mathrm{~kg}^{-1} \mathrm{~K}^{-1}$ and $C=6.05 \times 10^{26} \mathrm{Nm}^{-2}$.

Darcy's law used to predict the flow in the porous medium was modified to account for the inertia and boundary effects. The modification was described in a separate study [14].

\section{EXPERIMENTAL}

The $89 \mathrm{~mm}\left(3.5^{\prime \prime}\right)$ cube was to be heated in the isothermal oven at $200^{\circ} \mathrm{C}$ until thermal runaway was observed. The anisotropy of wood and geometry of wood cube was shown diagrammatically as in Fig.s 2 and 3. To study spontaneous heating, the wood cube was inserted with thermocouples type-T at the centre of the cube and connected to a data logger Yokogawa DAQ station DX230. If the centre temperature exhibited thermal runaway, the temperature would show a "jump" on the thermocouple readings. Thermocouples were positioned along longitudinal axis of the cube, the line connecting the end grain surfaces were denoted as $\mathrm{CH} 10, \mathrm{CH} 11, \mathrm{CH} 12, \mathrm{CH} 13, \mathrm{CH} 14, \mathrm{CH} 15, \mathrm{CH} 16$ and $\mathrm{CH} 17$. Thermocouple CH18 measured the oven air temperature. 


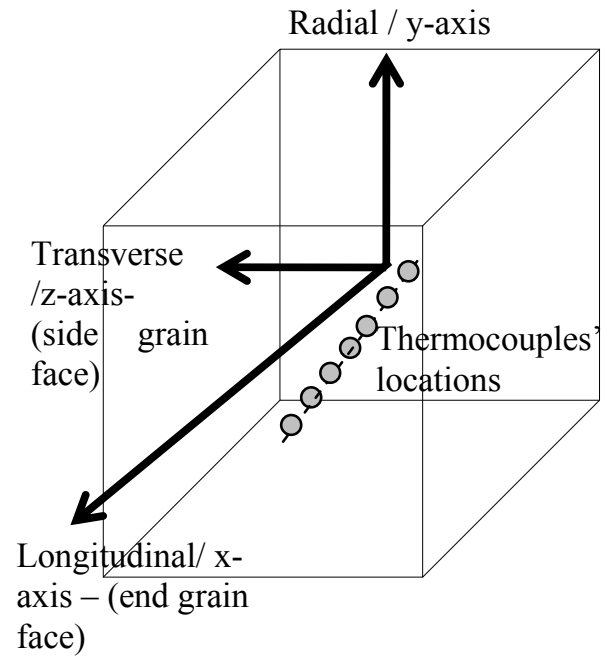

Fig. 2. The three principal axes of flow in wood cube

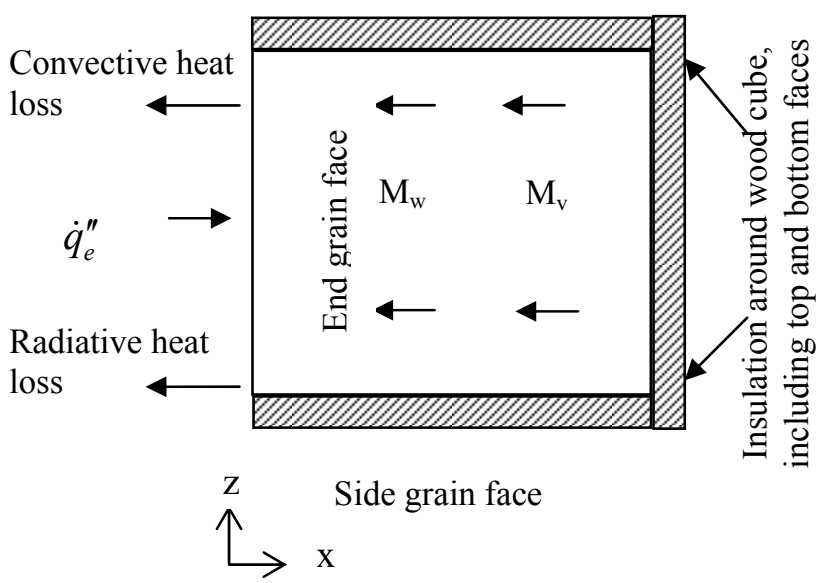

Fig. 3. Schematic domain on plan view

\section{RESULTS AND DISCUSSION}

\section{Velocity and temperature distribution of free water for initial heating}

To examine the velocity and temperature contours, contours at three locations measured from the mid-plane $(\mathrm{z}=0 \mathrm{~mm})$ were generated. The contours showed the results of the convective flows and temperature field associated with the longitudinal flow in the domain. The contours at the $x$ plane representing the transverse flows were not discussed since the flows were negligible in the transverse directions. For free water movement, because the model considers evaporation as the boundary condition, instead of an internal term, the velocity contours of free water did not exhibit large variation (see Figs. 4 to 6 ). The velocity contour near the exit (Fig. 6) however achieved more uniformity than any other inner location, which implied that evaporation occurred at the surface and vapour convected without resistance at the boundary in the computational domain. These results would only be possible if the liquid water content in those domains did not vaporise too quickly. The simulated free water movement agreed well with the phenomenological understanding of the initial drying stage of wood [15] where evaporation takes place almost entirely at the surface, and no retreating evaporation front has yet been occurred within wood domain.

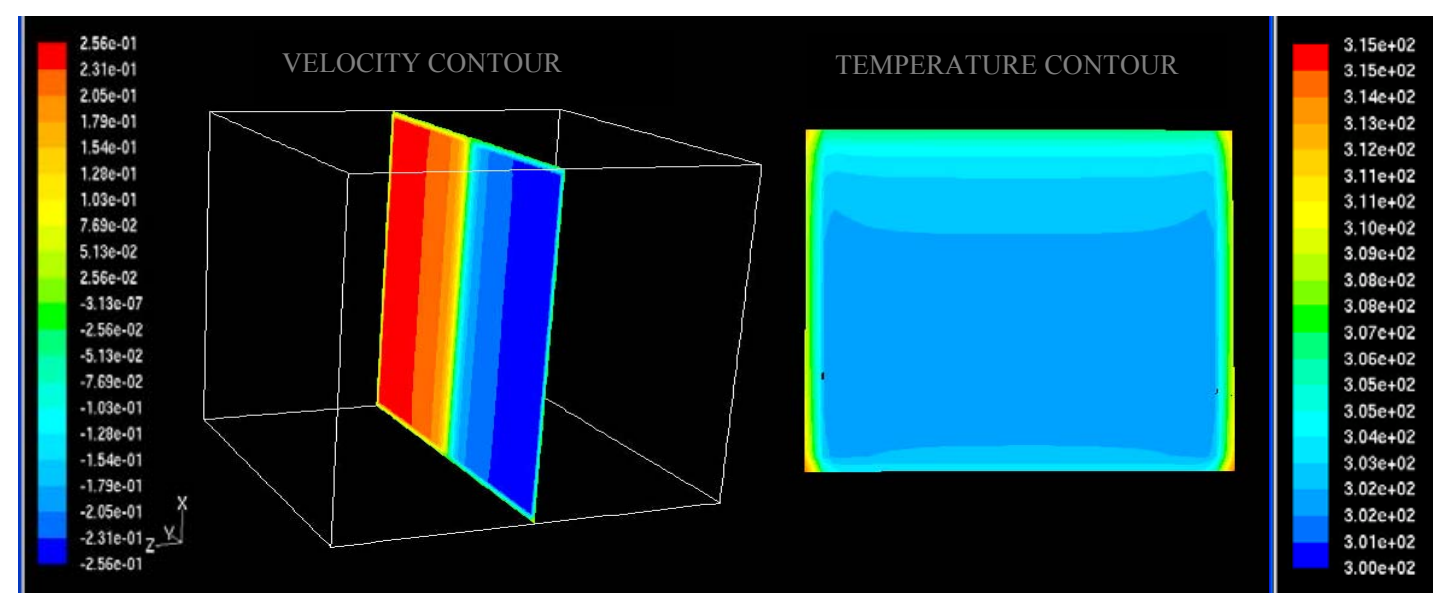

Fig. 4. Velocity and temperature contours on the mid-plane $(\mathrm{z}=0 \mathrm{~mm})$ for longitudinal flow 


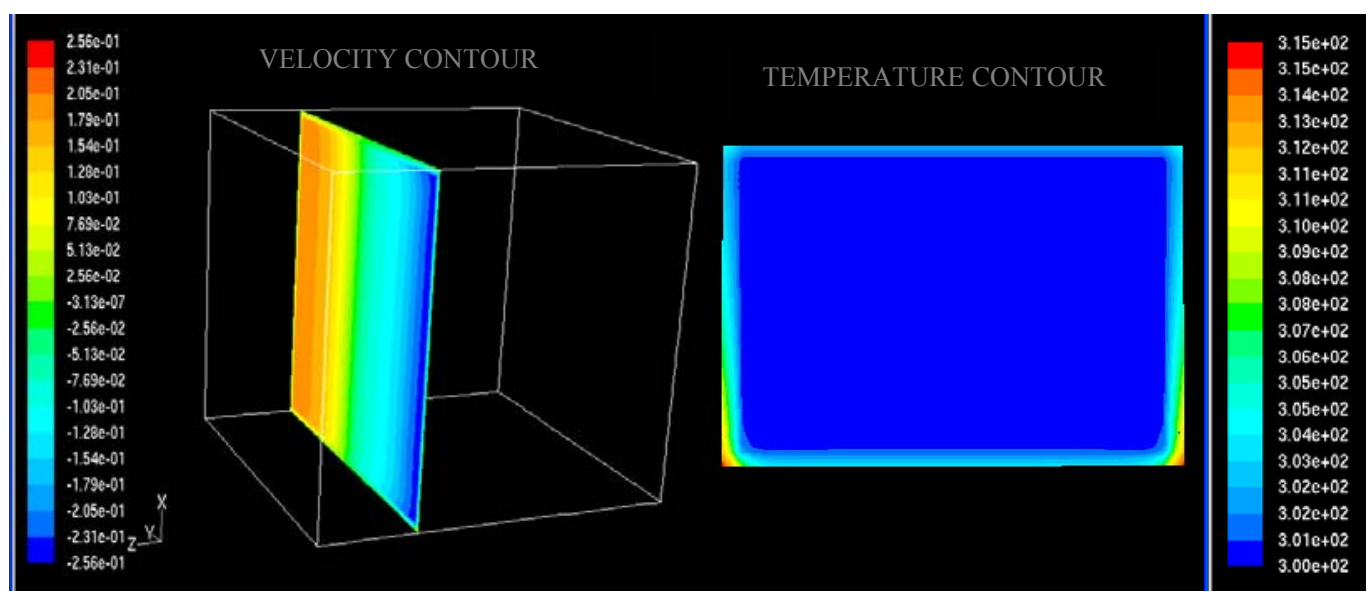

Fig. 5. Velocity and temperature contours on $z=20 \mathrm{~mm}$ from the mid-plane for longitudinal flow

The uniform temperature profile was the consequence of eliminating an internal evaporation term. To illustrate the effect, temperature profile generated from the modified Darcy's law Model was compared to the other two model's predictions from ref [12] for initial drying phase, where one was based on conventional drying model that considered evaporation as an internal term (Model 1), and the other simply as a diffusion model treating evaporation as a boundary condition (Model 2), the latter being just as the same as the modified model presented in this study. The parameters were tabulated in Table 1. As can be seen from Fig. 7, the temperature profile of Model 1 differed from Model 2 and the modified Darcy's law Model. Model 1 has a depressed curve while the temperature profiles of Model 2 and modified Darcy's law Model were rather linear. The linear temperature profiles of Model 2 and modified Darcy's law Model showed that the internal temperature distribution within wood volume was largely uniform.

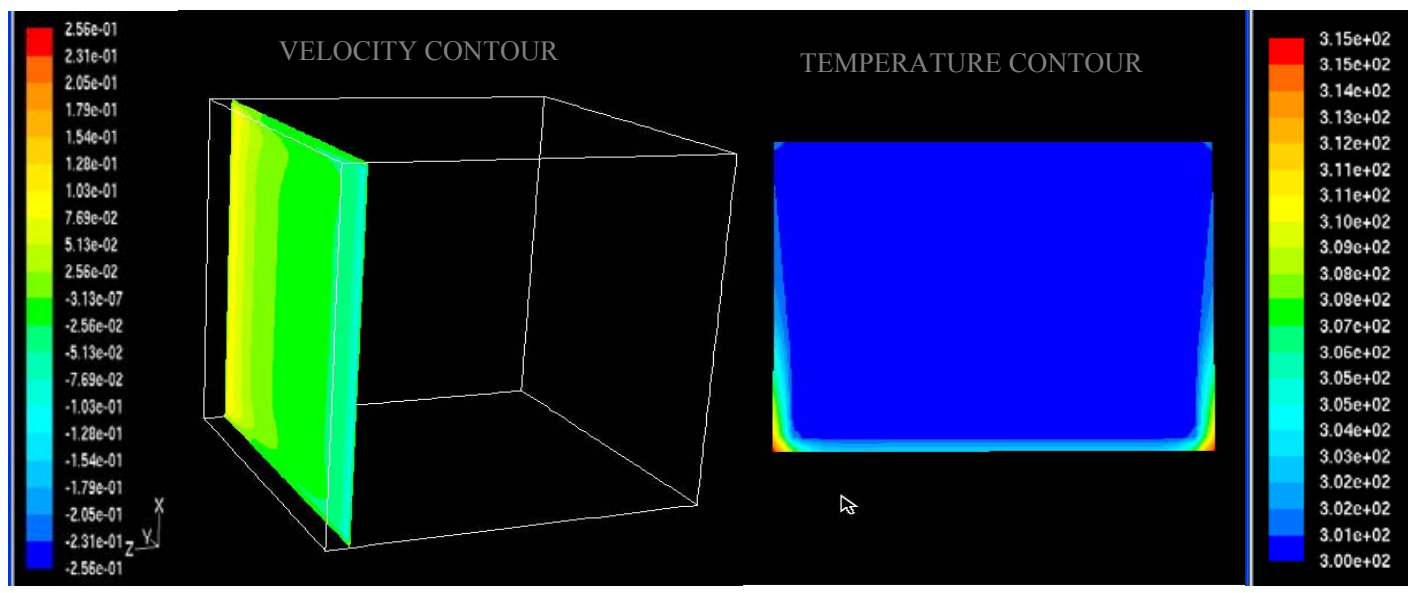

Fig. 6. Velocity and temperature contours near wall from the mid-plane for longitudinal flow

Table. 1. Parameters used in drying of wet wood for the initial period

\begin{tabular}{lcc}
\hline Item & Model 1 \& 2 & Modified Darcy's Law Model \\
\hline Initial water content $\left(\mathrm{kg} \mathrm{H} \mathrm{H}_{2} \mathrm{O} / \mathrm{kg}\right.$ dry wood) & 1 & 1 \\
Initial temperature $\left({ }^{\circ} \mathrm{C}\right)$ & 27 & 25 \\
Half thickness of wood $(\mathrm{m})$ & 0.01 & 0.01 \\
Liquid water diffusivity $\left(\mathrm{m}^{2} / \mathrm{s}\right)$ & $1.54 \times 10^{-9}$ & 1.45 \\
Density of wood $\left(\mathrm{kg} / \mathrm{m}^{3}\right)$ & 500 & 735.50
\end{tabular}




\begin{tabular}{lcc} 
Thermal conductivity of wood $(\mathrm{W} / \mathrm{mK})$ & 0.45 & 0.125 \\
Specific heat of wood $(\mathrm{J} / \mathrm{kgK})$ & 1405 & 2040 \\
Heating air temperature $\left({ }^{\circ} \mathrm{C}\right)$ & 82 & 200 \\
Vapour density in heating air $\left(\mathrm{kg} / \mathrm{m}^{3}\right)$ & 0 & 0 \\
Convective heat transfer coefficient $(\mathrm{W} / \mathrm{mK})$ & 22.5 & 20 \\
Convective mass transfer coefficient $(\mathrm{m} / \mathrm{s})$ & 0.0022 & 0.0022 \\
\hline
\end{tabular}

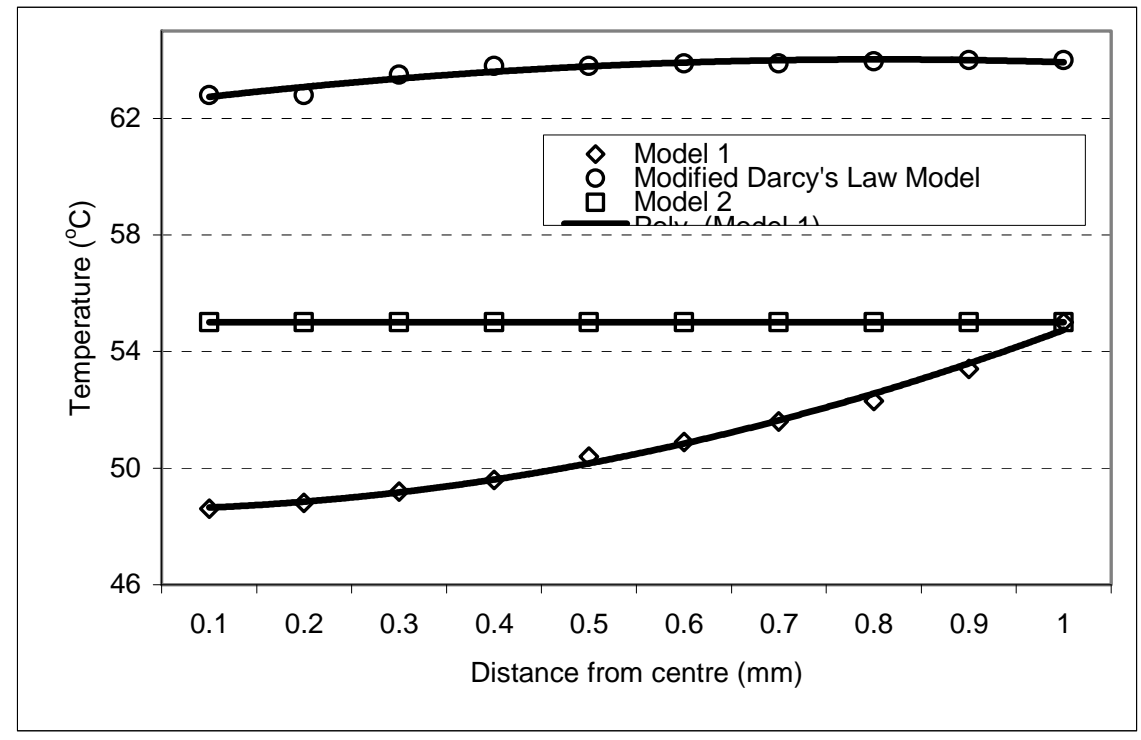

Fig. 7. Comparison of temperature profiles with different evaporation approaches

From the phenomenological point of view, the uniform temperature profiles were possible because firstly, evaporation took place at the surface and therefore only consumed heat from the ambient hot air; secondly the thermal conductivity was relatively large due to the considerable presence of liquid water at the low temperature drying stage. For Model 1 which considered evaporation as an internal term, heat was collected from inside the material and thus created a negative heat source inside. The system generated a temperature gradient to draw heat flux from the surface to the inside of the material. The inclusion of evaporation as an internal term created a heat sink in the system, resulting in the premature formation of a depressed temperature curve.

Another finding of consequential importance from the simulations is the direct link observed between velocity contours and the temperature profiles. The convective flows of free water within the domain were rather stable, hitherto uniform temperature fields. The temperature contours were shown besides the corresponding velocity contours in Figs. 4 to 6 . The consistent velocity contours and the correspondingly uniform temperature field offered useful insights in the development of self-heating examined later in this study.

\section{Velocity and temperature contours of moisture movement for extended heating}

Combined moisture (water and water vapour) has more pronounced convective flow pattern as compared to free water convection (Figs. 8 to 10). At this late drying stage, water flux inside the wood decreased. Evaporation front recessed from the surface and retreated inwards [16]. Evaporation was now taking place throughout the material and occurring as in the same amount as the mass change rate of the total moisture [17]. The flows within the domain should become much more erratic as compared to the movement of free water.

The temperature field also became highly non-uniform as a consequence of higher speed of water vapour convection within the domain. Unlike the initial drying stage where the temperature within the domain was 
rather uniform, the temperature field arising from combined moisture convection was more varied in tandem with the stronger convective flow. The model showed that the surface zone has a higher temperature. Diffusivity of liquid became small due to diminishing moisture content [18]. Less water flux diffused through the surface and the surface became drier and therefore hotter. The model therefore suggested an advancing temperature front towards the center. This result provided two crucial explanations on spontaneous ignition of wood experiment: firstly, when a porous mass of oxidizable material was considered for exothermic reaction, local variations in water content throughout the volume increased its propensity to spontaneous ignition by self-heating mechanisms that would not otherwise operate if it were at uniform water content. The retreating evaporation front also accounted for a characteristic S-curve temperature profile observable in the progression of self-heating in wood slab.

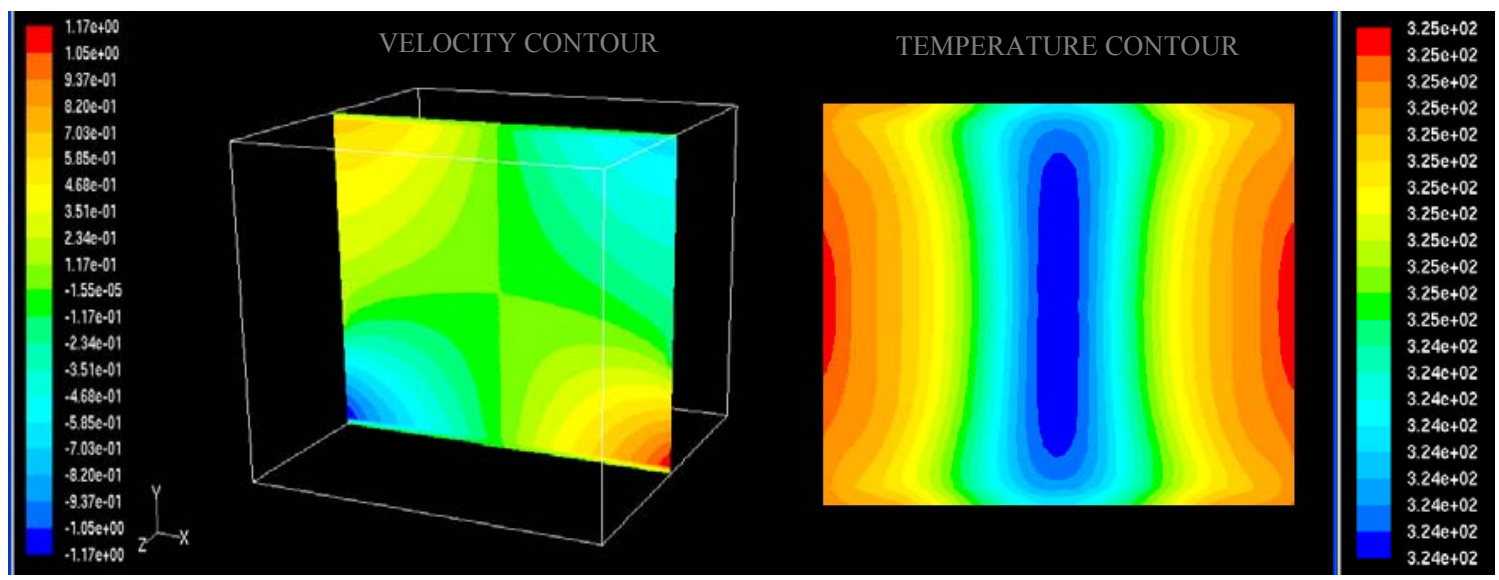

Fig. 8. Velocity and temperature contours on the mid-plane $(\mathrm{z}=0 \mathrm{~mm})$ for longitudinal flow

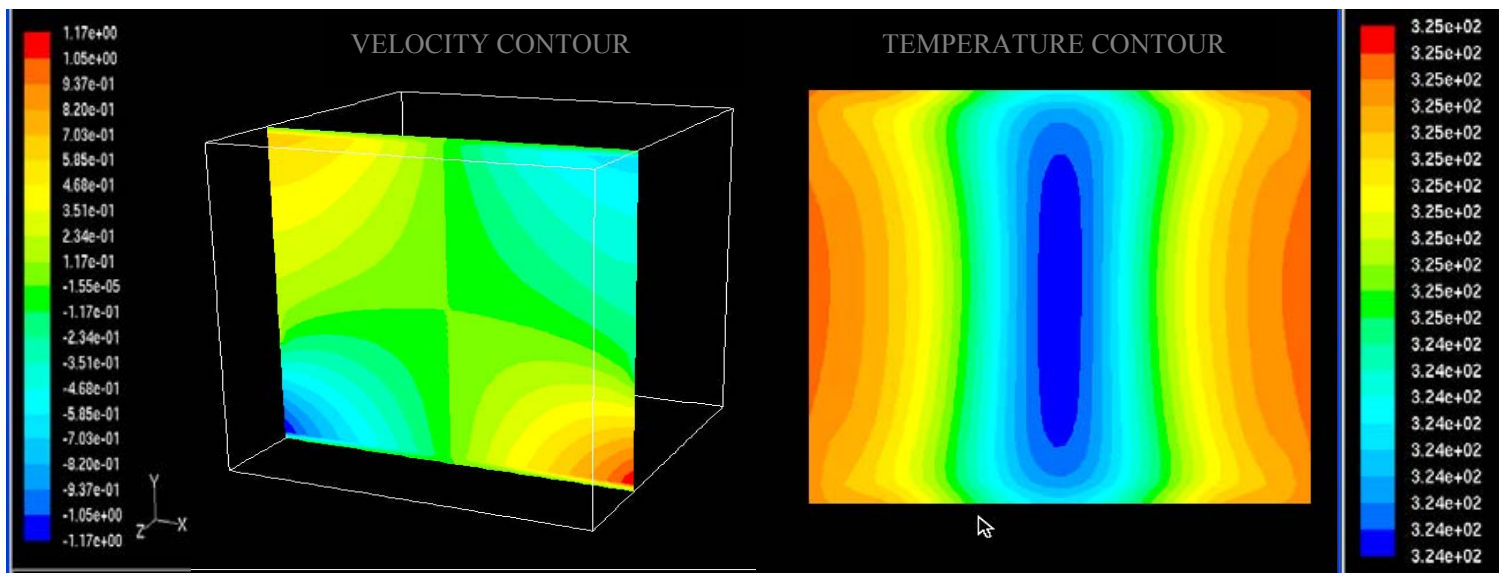

Fig. 9. Velocity and temperature contours on $z=20 \mathrm{~mm}$ from mid-plane for longitudinal flow 


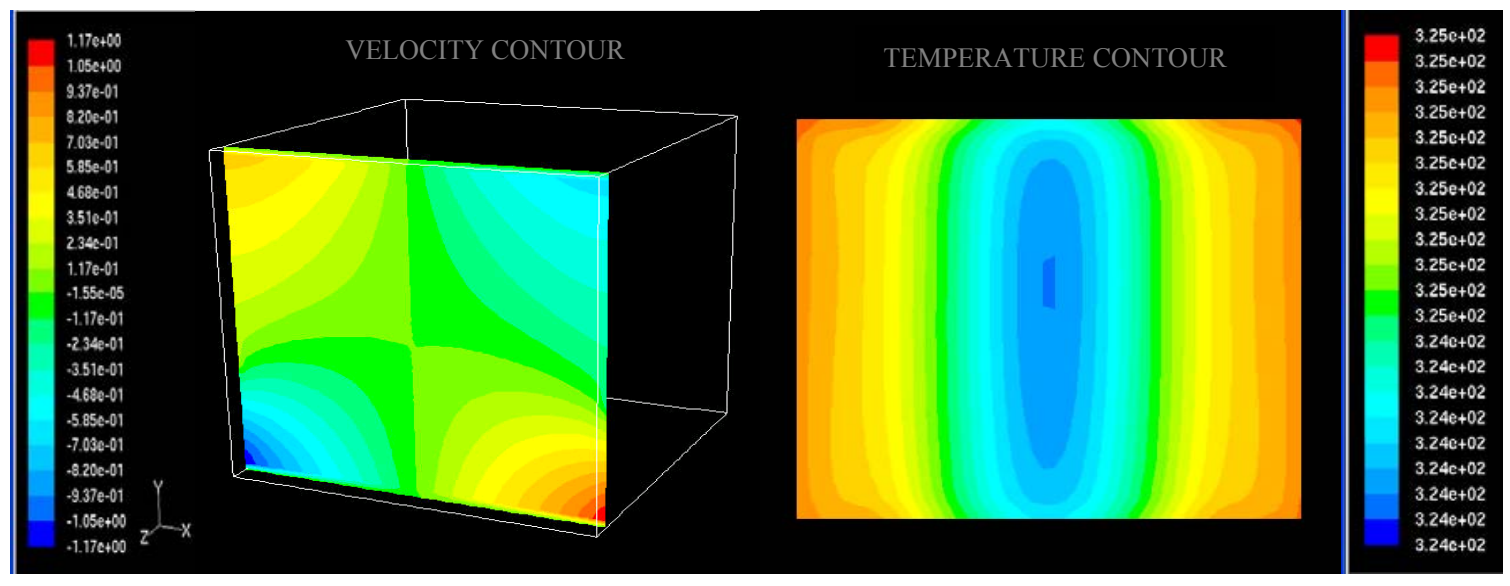

Fig. 10. Velocity and temperature contours near wall from mid-plane for longitudinal flow

Free water movement in the initial heating phase did not promote temperature-field development, lest in resulting in rapid temperature rise leading to thermal runaway. Only when the coupled velocity and temperature field commenced, The temperature profiles measured in wood cube, as shown in Fig. 11 and the lowest plot $(\mathrm{t}=30 \mathrm{~min})$ in Fig. 12 showed a characteristic $\mathrm{S}$ temperature-time curve, where the temperature was higher at the surface than the inner core. The temperature maxima at the surfaces started to move towards the centre. These dual maxima joined to form a single central maximum, raising the centre temperature of the wood cube. It was evident from Fig. 12 that self-heating became fully established once the centre temperature exceeded the surface temperature. The middle upper plot (time $=390 \mathrm{~min}$ ) showed the temperature right before self-heating dominated. The upper plot (time $=447 \mathrm{~min}$ ) indicated that selfheating has become fully established as the centre temperature reached the peak. The wood cube attained thermal runaway at the end of 9 hours.

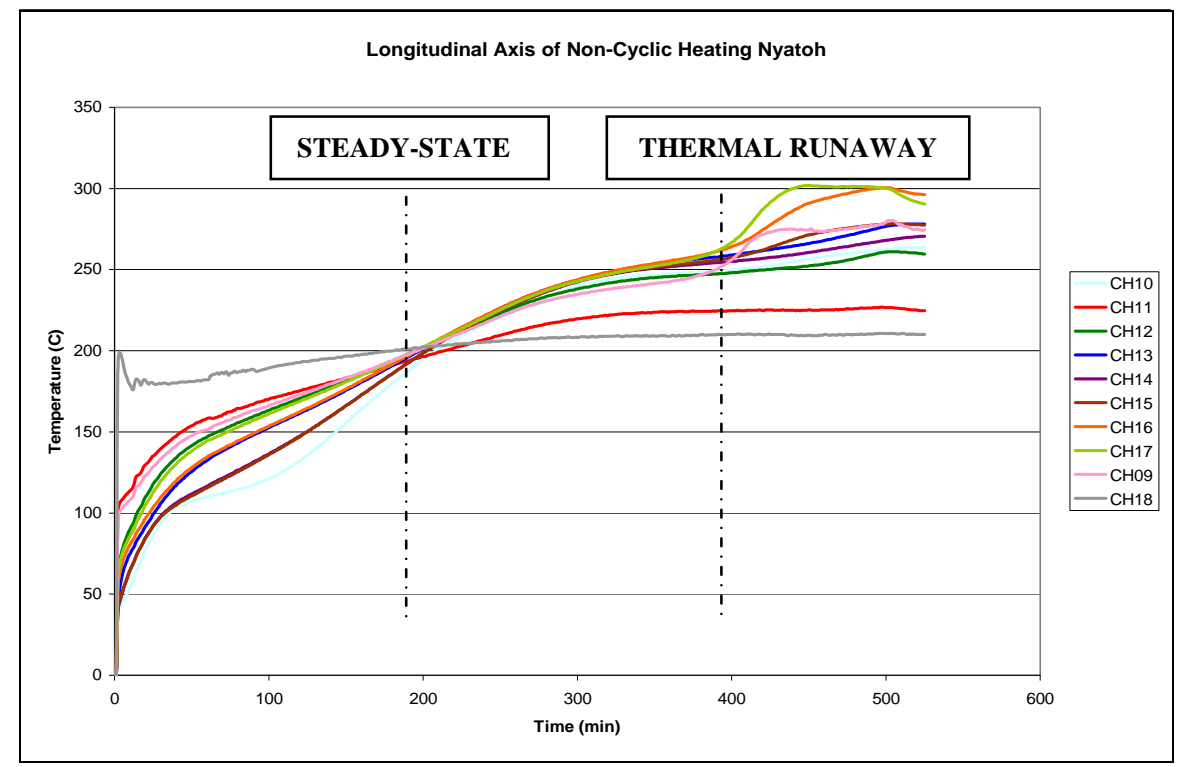

Fig. 11. Temperature-location profiles in longitudinal axis

\begin{tabular}{|l|l|}
\hline CH10, CH17: on end grain faces (EGF) & CH11, CH16: 6mm from EGF \\
\hline CH12, CH15: 13mm from EGF & CH13, CH14: 25mm from EGF \\
\hline CH09: 45mm i.e centre of cube & CH08: measuring oven air temperature \\
\hline
\end{tabular}




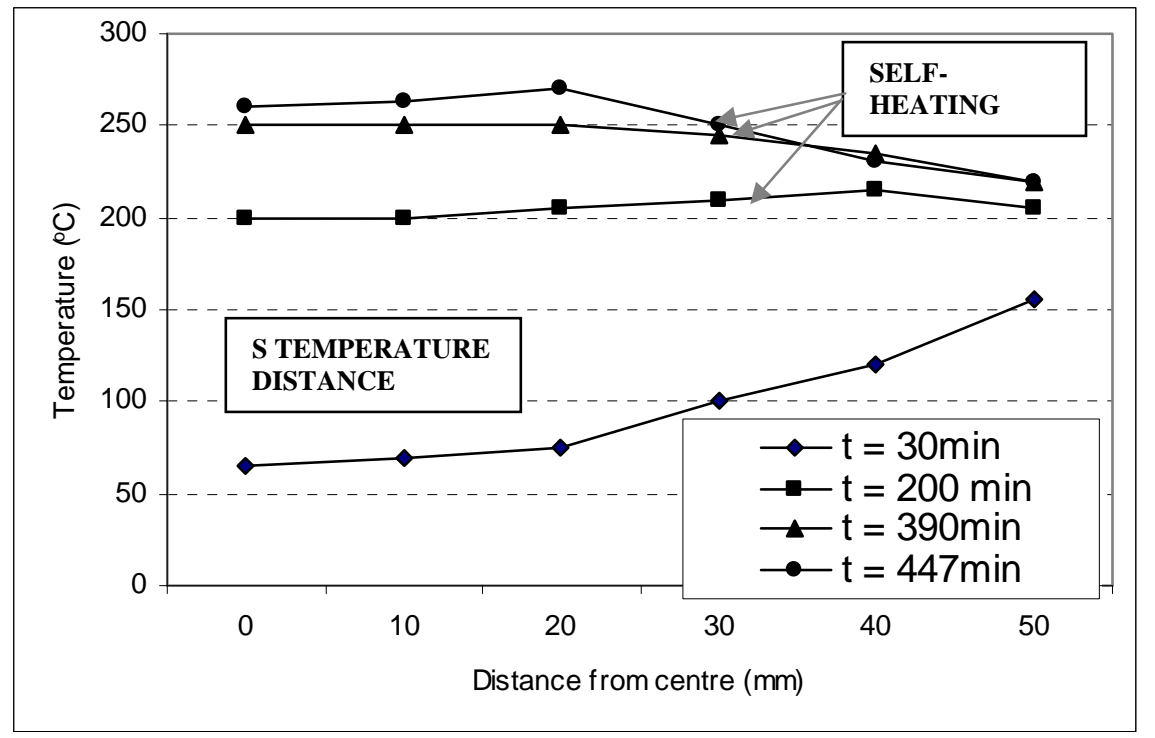

Fig. 12. S-temperature distance curve and self-heating

\section{CONCLUSIONS}

Self-heating and spontaneous combustion of wood was studied using wood cube of critical size exposed to low-temperature, long-term heating in an isothermal oven. A proposed porous model with modified Darcy's law has been set up to examine the interaction between fluid transport on temperature field development on low temperature drying in order to investigate the role of moisture transport on self-heating and spontaneous combustion of wood. The results showed that with surface evaporation incorporated into the initial phase model, a constant temperature distribution was obtained in tandem with a relatively stable free water movement within the domain. The extended heating phase using internal evaporation term demonstrated that combined moisture flow was stronger and erratic, bringing together a more distributed temperature profile. It was the coupled heat and mass transfer of combined moisture in the later that brought upon progression of self-heating in wood cube. The results therefore suggested that under constant heating regime i.e. isothermal heating exposure, liquid water movement alone might not promote selfheating. It was the internal evaporation where because of the coupled heat and mass transfer, initiated more rapid temperature field development.

\section{REFERENCES}

[1] Gatica, J. E., Viljoen, H. J. \& Hlavacek, V. (1989) Interaction between Chemical Reaction and Natural Convection in Porous Media. Chemical Engineering Science: 44, 1853-1870, doi:10.1016/0009-2509(89)85127-9

[2] Babrauskas, V. (2001) Pyrophoric Carbon...the Jury is Still Out. Fire and Arson Investigator: 51, 12-14.

[3] Fredlund, B. A Model for Heat and Mass Transfer in Timber Structures during Fire: A Theoretical, Numerical and Experimental study. Ph.D thesis, Department of Fire Safety Engineering. Sweden: Lund University, 1988.

[4] Alves, S. S. \& Figueiredo, J. L. (1989) A Model for Pyrolysis of Wet Wood. Chemical Engineering Science: 44, 2861-2869, doi:10.1016/0009-2509(89)85096-1

[5] Yuen, R. K.-K. Pyrolysis and Combustion of Wood in a Cone Calorimeter. Ph.D thesis, Mechanical Engineering, University of New South Wales, Australia, 1998.

[6] Perré, P., Moser, M. \& Martin, M. (1993) Advances in Transport Phenomena during Convective Drying with Superheated Steam and Moist Air. International Journal of Heat and Mass Transfer: 36, 2725-2746, doi:10.1016/0017-9310(93)90093-L 
[7] Krabbenhøft, K. Mositure Transport in Wood. A Study of Physical-mathematical Models and their Numerical Implementation. PhD thesis, Department of Civil Engineering. Lyngby: Technical University of Denmark, 2003.

[8] Vortmeyer, D., Dietrich, K. \& Ring, K. (1974) Comparison of One- and Two-phase Model Predictions for Adiabatic Packed-bed Chemical Reactors. Advances in Chemistry Series: 133, 588599.

[9] Claesson, J. \& Gaffner, D.. Moisture in Porous Building Materials, Sweden: Lund Institute of Science and Technology, 1977.

[10] Lianbai, G. \& Ping, M. Modelling of Water Transfer of Mason Pine Lumber during High Temperature Drying. 8th International IUFRO Wood Drying Conference, 2003, pp.24-29.

[11] Ping, M. \& Lianbai, G. (2003) Water Transfer of Mason Pine Lumber during High Temperature Drying. Holz als Roh-und Werstoff, 61, 349-354.

[12] Zhang, J. \& Datta, A. K. (2004) Some Considerations in Modelling of Moisture Transport in Heating of Hygroscopic Materials. Drying Technology: 22, 1983-2008, doi:10.1081/DRT200032740

[13] Sahota, M. S. \& P. J. Pagni (1979) Heat and Mass Transfer in Porous Media subject to Fires. International Journal of Heat and Mass Transfer: 22, 1069-1081.

[14] Lim, S.M \& Chew, M.Y.L. Interaction between Natural Convection and Chemical Reaction in Spontaneous Ignition of Solids. Interflam-Proceedings of the $11^{\text {th }}$ International Conference on Fire Science and Engineering, 2007.

[15] Fang, G. \& Ward, C. (1999) Examination of the Statistical Rate Theory Expression for Liquid Evaporation Rates. Physical Review E: 59, 441-453, doi:10.1103/PhysRevE.59.441

[16] Hukka, A. (1996) A Mathematical Model for Simulation of Softwood Drying in Temperatures above Boiling Point of Water with Special Attention to Boundary Conditions. Drying Technology: 14, 1719-1732, doi:10.1080/07373939608917169

[17] Eriksson, J., Johansson, H. \& Danvind, J. (2007) A Mass Transport Model for Drying Wood under Isothermal Conditions. Drying Technology: 25, 433-439, doi:10.1080/07373930601183785

[18] Plumb, O., Spolek, G. \& Olmstead, B. (1985) Heat and Mass Transfer in Wood during Drying. International Journal of Heat and Mass Transfer: 28, 1669-1678. 